\title{
Direct fabrication of silicone lenses with 3D printed parts
}

\author{
Tahseen Kamal ${ }^{\mathrm{a}}$, Rachel Watkins ${ }^{\mathrm{a}}$, Zijian Cen ${ }^{\mathrm{a}}$, W. M. Lee ${ }^{* \mathrm{a}}$ \\ ${ }^{a}$ Research School of Engineering, College of Engineering and Computer Science, Australian \\ National University, North Road, ACT 2601, Australia.
}

\begin{abstract}
The traditional process of making glass lenses requires grinding and polishing of the material which is a tedious and sensitive process. Existing polymer lens making techniques, such as high temperature reflow techniques, have been significantly simple lens making processes which cater well to customer industry. Recently, the use of UV-curing liquid lens has ushered in customized lens making (Printed Optics), but contains undesirable yellowing effects. Polydimethylsiloxane (PDMS) is a transparent polymer curable at low temperature $\left(<100^{\circ} \mathrm{C}\right)$ provides an alternative to lens making. In this work, we showed that PDMS lenses are fabricated using single silicone droplets which are formed in a guided and controlled passive manner using 3D printed tools. These silicone lenses have attributes such as smoothness of curvature, resilience to temperature change, low optical aberrations, high transparency (>95\%) and minimal aging (yellowing). Moreover, these lenses have a range of focal lengths (3.5 $\mathrm{mm}$ to $14.5 \mathrm{~mm}$ as well as magnifications (up to $160 \mathrm{X}$ ). In addition, we created smartphone attachment to turn smart device (tablet or smartphone) into a low-powered microscope. In future we plan to extend this method to produce microlens array.
\end{abstract}

Keywords: Passive, silicone droplet, polymer, silicone lens, smart device microscope.

\section{INTRODUCTION}

Lenses form an important element of almost all standard microscope systems. Lenses redirect light that reflects off/transmits through an object onto a secondary plane where a virtual copy of the object is reconstructed. Short focal length lenses (simple magnifying glass) create a larger copy (image) of the object that is relayed onto an imaging sensor. In refractive lenses, the focal length is intricately linked to the curvature of the lenses.

\subsection{Moldless fabrication of silicone lenses}

Recently, direct fabrication of silicone/polydimethylsiloxane (PDMS) lenses ${ }^{1-3}$ has been demonstrated using alternative high performing lenses. These PDMS lenses exhibit high refractive indices $(\mathrm{n}=1.39-1.55)$ and optical transparency (>95\%) within visible spectrum of the wavelength, without encountering issues of nominal aging (yellowing). More importantly, they can be shaped under low temperatures and are commonly resilient to high temperatures $\left(>125^{\circ} \mathrm{C}\right)$ as demonstrated in recent moldless techniques.

Moldless fabrication of lenses relies on the formation of liquid droplets, which is the interplay of viscous drag, surface tension, capillary forces and gravitational forces. In this work, we describe a stable passive droplet dispenser and lens curing holder. The combination creates static pendant drops which are then harvested as optical lenses. Previous droplet lens manufacturing techniques uses active droplet dispensers (syringes, needles) ${ }^{3,4}$ hat dispenses droplets of specific volume. While the approach has its advantages in accurate volume being dispensed, it requires a plunger devices i.e. syringes that adds a layer of complexity into the fabrication process i.e. plunger and container assembly. In the following section, we describe a fully passive dispenser that aims to recapitulate the natural formation of droplets ${ }^{5}$ from drippingdrops. In the way, the entire lens making device can be directly printed without any need for assembly and can be easily replicated by any individual.

\section{SILICONE LENS HARVESTING PROCESS}

In this work silicone droplet lens fabrication has been done using a set of 3D printed substrates. Three parts were designed using Solidworks $@$ and were printed using a 3D-UP Mini printer which are: (i) the basins, to hold the PDMS

SPIE BioPhotonics Australasia, edited by Mark R. Hutchinson, Ewa M. Goldys, Proc. of SPIE Vol. 10013, $1001336 \cdot$ @ 2016 SPIE · CCC code: 0277-786X/16/\$18 · doi: 10.1117/12.2242967 
mixture, (ii) the droplet-holders with holes to hold the droplets and (iii) the droppers that are used to extract PDMS mixture and allow the droplets to be formed and dripped onto the holder. Figure 1a shows the 3 parts.

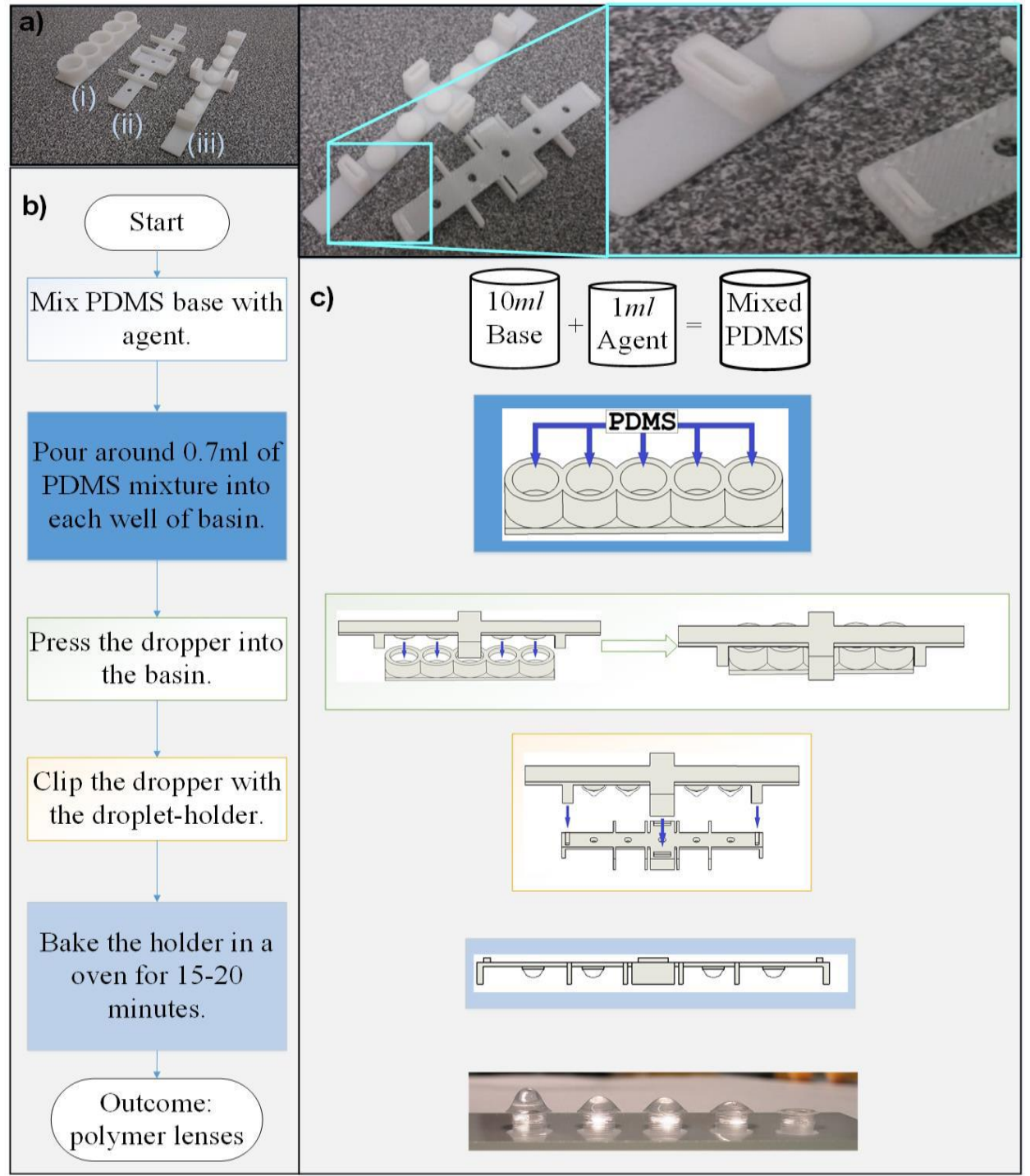

Figure 1: PDMS lens making process using 3D printed substrates which uses the natural phenomenon of Rayleigh breakup to form droplets. (a) The three lens making kits, (i) Basin, (ii) Droplet-holder, (iii) Conic Droppers. Clipping attachment with the dropper and the holder to ensure better alignment. (b)-(c) Lens making process using the kits.

The step-by-step procedure to fabricate the lenses using the 3D printed parts is illustrated in a flowchart, in Figure 1b-1c. Firstly, a PDMS base agent is mixed with the curing agent with a ratio of 10:1 for approximately 3-4 minutes. After which, the solution is degassed (60 minutes- desiccator, 20-30 minutes -vacuum pump) to remove air bubbles. A regular $3 \mathrm{ml}$ plastic syringe then extracts a small amount of PDMS $(\sim 0.7 \mathrm{ml})$ that fills each basin (i). The droppers are then pressed into the holes of the basin to extract PDMS droplets as shown in Figure 1c. The droppers are placed on the droplet-holder using a self-aligning mechanical clip which is used to ensure that each dropper is aligned and positioned at a desired distance over each hole and a misalignment of up to $\sim 10 \%$ will lead to successful transfer of the droplet. Depending on the viscosity of the mixed PDMS, it takes about 15-20 seconds for the droplets to be dispensed onto the droplet holder. Once the droplets are formed on the droplet-holder, the holder is placed in the oven at $70^{\circ} \mathrm{C}$ for 15 minutes. After curing, the lenses are then removed from the droplet holder. The 3D printed kits can be re-used. This passive dispenser approach to lens-making has significant advancement from the previously published work ${ }^{1}$ [15] which 
was prone to misalignment error during inversion process with a very low throughput of around $25 \%$ whereas the new approach yields $97 \%$ throughput. The throughput here has been considered as successful lenses with high image quality and shape conformity.

\section{DROPLET GENERATION USING PASSIVE DISPENSER}

The phenomenon of droplet formation from a flowing liquid jet has been observed by Plateau and later Lord Rayleigh elaborated that Rayleigh breakup occurs on a fluid depending on the viscosity, length and radius of the jet ${ }^{6}$. The liquid flowing as a jet will break up into various sized liquid droplets if there is instability or will form a single droplet if the jet is stable. Based on this concept a set of 3D printed kit has been designed to generate liquid droplets at the tip of a conic shaped substrate.

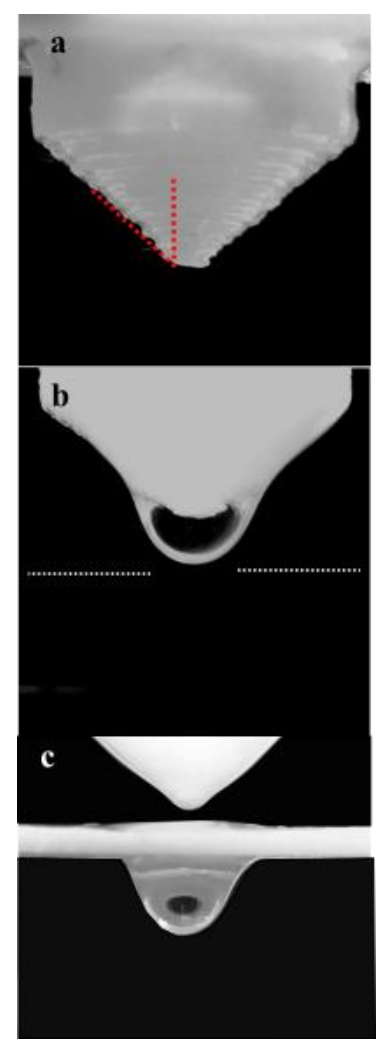

Figure 2: Droplet formation using the passive dispenser. (a) The tip of conic dropper showing the angle of $50.1^{\circ}$, (b) Droplet formed at the tip of the dropper, (c) Droplet transferred from the tip to the holder.

In Figure $2 \mathrm{a}$ the conic dropper has been shown imaged using a simple imaging system ( $45 \mathrm{~mm}$ lens, Raspberry PI camera). In Figure $2 \mathrm{~b}$ the dropper has a droplet formed at the tip after immersion. In conventional dispensing, using a syringe, a slowly moving liquid grows a drop at the end of a nozzle of syringe. As the drop grows in size, the weight of the droplet exceeds the surface tension and capillary force where a discrete droplet is formed. In the nozzle scenario, a small amount of pressure is usually applied at the distal end of the nozzle to initiate this droplet growing process. The experimental setup of using an active dispenser (such as a syringe) to generate droplets requires complex setup. Instead, here we propose the use of cone-shaped substrate to produce a dominant gravitational force i.e. large bond number $G$, $G=\frac{\rho R^{2} g}{\sigma}$, where $R$ is the radius of curvature, $\sigma$ is the surface tension, $\rho$ is the density of the liquid and $g$ is the gravitational acceleration. A large $G$ value ( $>1)$ indicates that the gravitational force dominates over surface tension necessary to form a spherical droplet. Another purpose of the cone shape is to provide adequate surface area so that there is sufficient capillary forces $(>1 \mathrm{~mm})$ to extract a certain amount of liquid and ensure that the extracted drop is close to 
the critical mass to create the dripping process ${ }^{7}$. Based on the critical mass, $m_{c}=3.8 \frac{\sigma R}{g}(\sim 0.032 \mathrm{~g})$, the cone radius is designed with a base radius of $5 \mathrm{~mm}$ and slope of $50.1^{\circ}$.

While a jet flows the breakup occurs following a sinusoidal pattern of wavelength $\lambda$. If $\lambda$ is higher than optimum then Rayleigh-Plateau instability occurs and a series of satellite droplets form following the primary droplet. To ensure smooth transfer of the droplet from the tip of the dropper to the holder the height between the tip and the holder has been designed deliberately to be smaller than the optimum wavelength ${ }^{8}, \lambda_{\text {opt }}=2 \sqrt{2} \pi r$, where $r$ is the radius of the jet. Keeping the height minimum $(0.5 \mathrm{~mm})$ ensures that the primary droplet is transferred and no satellite droplet can transfer to the holder.

\section{CHARACTERIZATION OF THE SILICONE LENSES}

In order to verify the performance of the lenses harvested using the passive dispenser a series of experiments has been to observe the optical and physical properties of the lenses. Here we summarize the results and the analysis.

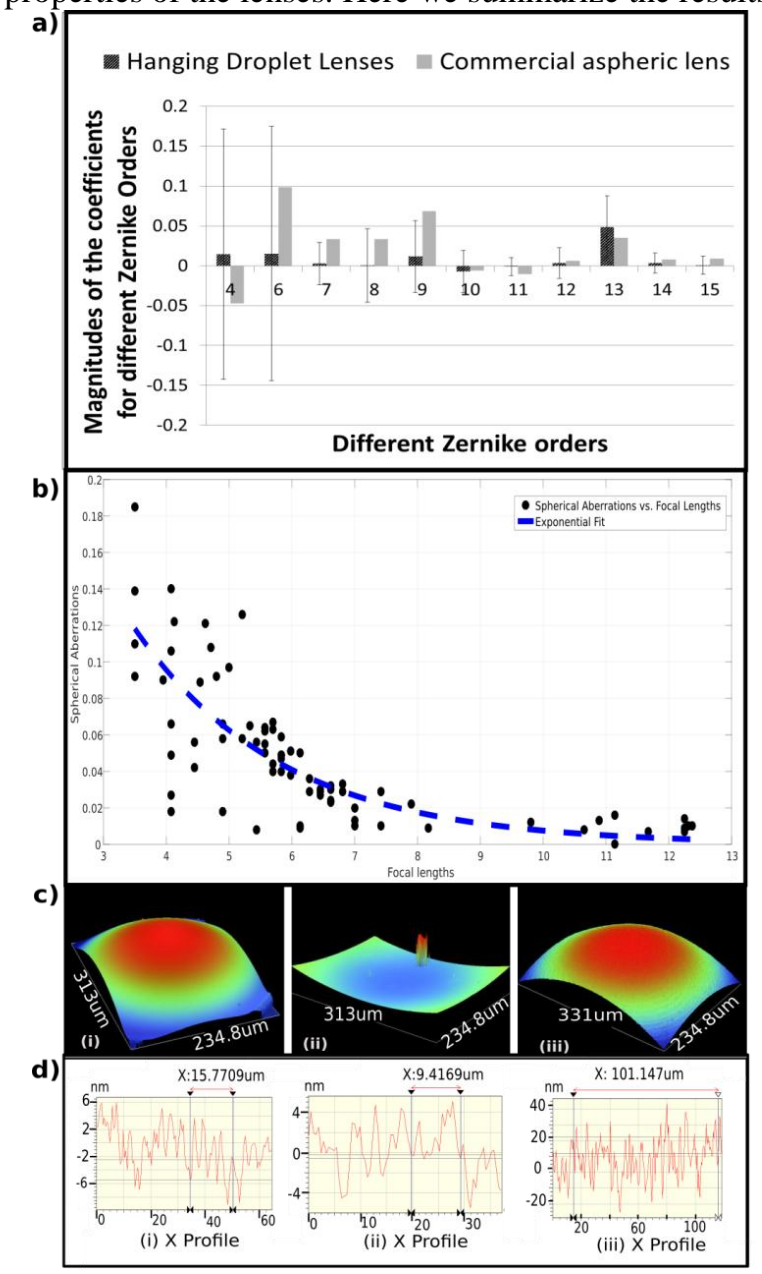

Figure 3: Optical and physical characteristics of the lenses (a) Optical aberrations present in the droplet lenses compared to a commercial aspheric lens, (b) Due to the presence of high spherical aberrations, another study has been made to observe the relationship between focal lengths and the spherical aberrations. The plot summarizes the correlation. (c) 3D maps of surfaces of silicone lenses and the aspheric lens. (d) Line plot for a section of the 3D map showing small surface roughness of the silicone lenses. 


\subsection{Optical characteristics}

Droplet lenses produced using our approach have a range of magnifications. The degree of convexity is related to the focal length or magnification. Following the lens makers formula, modified for plano-convex lenses, $\frac{1}{f}=(n-1)\left(\frac{1}{R_{1}}-\frac{1}{R_{2}}\right)$, it can be observed that the high convex lenses have higher magnification capability. To measure the aberrations of the lenses an optical setup using a Shack-Hartmann wavefront sensor has been used. The aberrations found have been compared with a commercially available aspheric lens as shown in Figure 3a. It has been found the silicone lenses have compatible optical aberrations with the commercial lens. Zernike mode 13 corresponds to the spherical aberration which has been empirically found to be high in the droplet lenses. Further experimentation has been done to establish a correlation between the focal lengths and spherical aberrations which has been summarized in Figure $3 b$.

\subsection{Physical characteristics}

During curing, it is also possible that the delicate liquid air interface of droplet formation can introduce uneven surfaces on the silicone droplets ${ }^{8}$. To study that surface roughness of the lenses were measured using an optical profiler (Wyko NT9100: Mirau interferometer) and compared against commercial aspheric lenses. The results, as shown in Figure $5 \mathrm{a}$ and $5 \mathrm{~b}$, in the form of the 3D maps (Figure 3c) and a cross-sectional plot (Figure 3d), suggest that the silicone lenses have a highly conforming surface. The measurements suggest that the silicone lenses possess lower magnitude of surface roughness compared to the commercial aspheric lens. The RMS value of the surface roughness of the commercial aspheric lens was found to be $15 \mathrm{~nm}$ and for the silicone lenses harvested using the passive dispenser was found to be $3.21 \mathrm{~nm}$ for the front surface and $1.91 \mathrm{~nm}$ for the back surface.

\section{IMAGING USING THE SILICONE LENSES}

The silicone lenses can be attached to any smart device using a 3D printed clip ${ }^{9}$ that can turn the smart device into a handheld microscope. Figure $4 \mathrm{a}, 4 \mathrm{~b}$ show the The imaging performance of different lenses has been observed using a simple imaging setup of a Raspberry PI camera, LED and droplet lenses. The achieved magnifications were up to 160X. The various resolutions achieved from the different lenses ranged from $300 \mu \mathrm{m}$ to $\sim 3.14 \mu \mathrm{m}$. Figure 4 shows the resolutions achieved using three different lenses and a commercial aspheric lens.

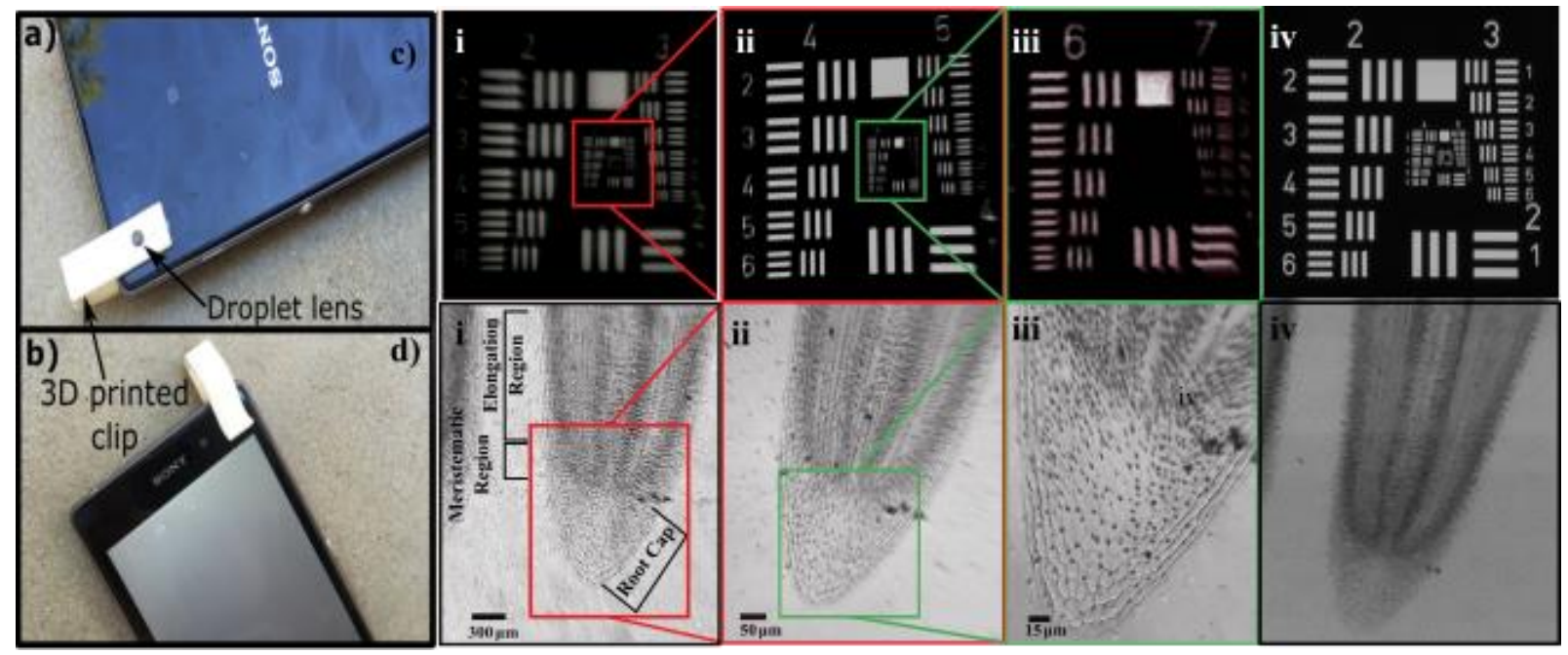

Figure 4: Imaging using the droplet lenses. (a)-(b) a 3D printed clip can be used to attach the droplet lenses with any smart device and use for imaging on demand. (c) A 1951 USAF target card has been imaged using various lenses. (i) A droplet lens of small curvature which showed similar performance as the aspheric lens. (ii) A droplet lens of medium curvature can resolve up to $50 \mu \mathrm{m}$ (iii) A droplet lens of high convexity can resolve up to element 
2 of group 7. (iv) A commercial aspheric lens of focal length $18.1 \mathrm{~mm}$ has been used to compare the imaging performance. (d) The same lenses have been used to image the tip of onion roots.

The droplet lenses of different curvature can be used to achieve different imaging performances. The quality of images of the droplet lenses is compatible with commercial aspheric lenses.

\section{CONCLUSION}

The use of soft polymer for the design of optical components has been done for decades. Using the concept of natural phenomenon of droplet formation and a set of 3D printed substrates a large scale of droplet lenses can be harvested. This process is low-cost, easily adaptable by someone who is interested and can produce quality optical lenses. The imaging performance can be improved by using computational techniques to remove aberrations ${ }^{10}$. In the future the concept can be enhanced to harvest multilens arrays.

\section{REFERENCES}

[1] W. M. Lee, A. Upadhya, P. J. Reece, and T. G. Phan, "Fabricating low cost and high performance elastomer lenses using hanging droplets," Biomedical Optics Express 5, 1626-1635 (2014).

[2] C. McDonald, and D. McGloin, "Low-cost optical manipulation using hanging droplets of PDMS," RSC Advances 5, 55561-55565 (2015).

[3] Y.-L. Sung, J. Jeang, C.-H. Lee, and W.-C. Shih, "Fabricating optical lenses by inkjet printing and heat-assisted in situ curing of polydimethylsiloxane for smartphone microscopy," Journal of Biomedical Optics. 20, 047005047005 (2015).

[4] M P. Vilmi, S. Varjo, R. Sliz, J. Hannuksela, and T. Fabritius, "Disposable optics for microscopy diagnostics," Scientific Reports. 5, 16957 (2015)

[5] Utada, L.-Y. Chu, A. Fernandez-Nieves, D. Link, C. Holtze, and D. Weitz, "Dripping, jetting, drops, and wetting: the magic of microfluidics," Mrs Bulletin. 32, 702-708 (2007)

[6] Strutt, J.W. Lord Rayleigh., "On the instability of jets," Proceedings of the London mathematical society 10, 413 (1878).

[7] S. D. R. Wilson, "The slow dripping of a viscous fluid,” Journal of Fluid Mechanics. 190, 561-570 (1988).

[8] J. A. Lock, J. D. Walker, and J. H. Andrews, "Using refraction caustics to monitor evaporation of liquid drop lenses," Applied Optics 29, 4599-4607 (1990).

[9] "PNNL Smartphone Microscope - Available Technologies - PNNL". at <http://availabletechnologies.pnnl.gov/technology.asp?id=393> (12 September 2016).

[10] G. Zheng, R. Horstmeyer, and C. Yang, "Wide-field, high-resolution Fourier ptychographic microscopy,". Nat Photon. 7, 739-745 (2013). 